\title{
SHORT IMPLANTS TO RESTORE MISSING TEETH IN SEVERE RIDGE DEFICIENCY IN PSTERIOR MAXILLARY REGION
}

\author{
Tarek Mohamed Abdelsamad*, Mohamed Abd-Elakher Omar** and Usama Abou El-Enein Mohamed ${ }^{* * * *}$
}

\begin{abstract}
The aim of this study was to evaluate clinically and radiographically the outcomes of using short implants as an alternative for sinus lift in extreme atrophic maxilla. Patients and methods: partially edentulous patients of both sexes (mean age 31.5 years, range 27.0-55.3 years, seven women and five men) were selected from the out-patient clinic, Faculty of Oral and Dental Medicine, Boys, Cairo, Al-Azhar University. The patients were divided into two groups: Group (I) the patients were treated with dental short implant placement without sinus lift, (eight implants with short length $5 \mathrm{~mm}$ ). Group (II) the patients were treated with dental implant placement after sinus lift, (four implants with long length which higher than bone 2-3 mm) according to case. Patient examination past medical history, past dental history, chief complaint). Radiographic examination (by panoramic X-ray \& Cone Bram C.T. Presurgery and Postsurgery 0,3,6 months). Surgical procedures for implant installation. After muco-periosteal flap was performed in the prepared surgical site, large round surgical bur (no. 6) rotating at very low-speed was introduced at the prospective implant site in the maxilla to create bony concavity which is necessary for stable direction control of Pilot drill. Then, the implant was slowly threaded into the bone using ratchet or handle instrument by a clockwise direction under external saline irrigation. Topical ointment antibiotic was applied, then fixture was covered after black retraction suture was removed, then thorough debridement and irrigation of the surgical field with sterile normal saline solution. The flap was repositioned and sutured. Methods of evaluation: all implants were tested for proper osseointegration, function and absence of mobility at three and six months after surgery. The condition of the gingival tissues around the fixture abutments was evaluated according to Loe and Sillness. Radiographic Evaluation: all subjects of the study were submitted to Cone Beam scanning before implant placement, another cone beam C.T. was taken immediately after placement, after 3 months and after 6 months for bone density measurements (densitometry analysis) and crestal bone resorption. Results: the mean value of crestal bone loss was 0.29 mm, with a maximum of $1.77 \mathrm{~mm}$. No significant differences were found in the rate of bone thickness loss between group (I) and group (II) (P=0.191). The mean value of bone loss increased in longer implants, but the difference was not significant ( $\mathrm{P}=0.313)$. Spearman's correlation coefficient was 0.039 between the mean value of bone loss and implant length $(\mathrm{P}=0.717)$ and 0.242 between the patients' age and implant length $(\mathrm{P}=0.068)$. On conclusion; I) Short implants' is successful treatment modality to avoid invasive surgical treatments at atrophic areas of posterior maxilla, such as bone grafts and sinus lifting. II) The reconstruction of edentulous, atrophic jaws according to functional and aesthetic factors not only restores chewing function, but leads to positive psychosocial effect and thus also improves the patient quality of life.
\end{abstract}

\section{INTRODUCTION}

The introduction of dental implants has led to a turning point in the rehabilitation of partially or totally edentulous patients ${ }^{(1)}$. However, not always, the placement of dental standard length implants is possible or feasible in the first instance. Several anatomical conditions affect the rehabilitation treatment as maxillary posterior regions ${ }^{(2)}$. Historically, clinical studies have reported on low survival rates of short implants $(\leq 10 \mathrm{~mm})$. These studies describe implants with machined surfaces, mostly placed in posterior regions with

\footnotetext{
* Professor of Oral and Maxillofacial Surgery Department, Faculty of Dental Medicine, Boys, Cairo, Al Azhar University ** Professor and Head of Oral and Maxillofacial Surgery Department, Faculty of Dental Medicine, Boys, Cairo, Al Azhar University *** B.D.S. (El-Mansura University), MSc (Al-Azhar University).
} 
higher loads and softer bone compared with more anterior regions ${ }^{(3)}$. Cinical studies on short implants with rough surfaces report survival rates similar to implants in general. Two extensive reviews concluded that the survival rates for short implants were found to be comparable with those obtained for longer implants placed under similar conditions, when using appropriate surgical technique and implants with a rough surface ${ }^{(4)}$. This is supported by some clinical studies on implants products where the survival rates for shorter implants (8-9 $\mathrm{mm})$ were similar to longer implants $(\geq 10 \mathrm{~mm})$ ${ }^{(5)}$. The current study is an attempt to assess and compare the final outcomes of dental short implant installation into atrophic maxilla vs. mucosal sinus membrane elevation.

\section{Anatomical consideration in posterior} maxilla for implant instelation for many dental practitioners, the edentulous posterior maxilla is considered a clinical challenge during dental implant treatment ${ }^{(6)}$. This is because its insufficient bone quality, deficient alveolar ridge, spiny ridges, undercuts, and sinus pneumatization are often encountered after tooth loss. Several approaches have been developed and are currently used to overcome these problems, two of them being sinus augmentation and bone augmentation ${ }^{(7)}$. Elevation of the maxillary sinus floor was first published by Boyne and James (1980) ${ }^{(8)}$. After these reports, several techniques were reported for successful sinus floor elevation, including crestal and transalveolar approaches. A crestal approach uses the osteotome technique introduced by Summers in $1994^{(9)}$. Dental practitioners used two main procedures of sinus floor elevation for dental implant placement: twostage technique using the lateral window approach, and a one-stage technique using a lateral or a crestal $\operatorname{approach}^{(10)}$.

\section{Sinus pneumatization and residual bone resorption}

Maxillary sinus pneumatization is a physiologic process that occurs in all Para nasal sinuses during the growth period, causing them to increase in volume $^{(11)}$. The reasons for sinus pneumatization are poorly understood, but factors that cause this process include heredity, the pneumatization drive of the nose's mucous membrane, craniofacial configuration, density of the bone, growth hormones, sinus air pressure, sinus surgery, and posterior tooth extraction ${ }^{(12)}$. According to a radiographic study, pneumatization was more significant after extraction of teeth enveloped by a superiorly curving sinus floor, extraction of several adjacent posterior teeth, and extraction of second molars as opposed to first molars ${ }^{(12)}$. Residual ridge resorption following tooth extraction is unavoidable process in posterior maxillary area. Extensive ridge resorption is one of the many problems for implant-prosthetic treatment in the posterior maxillae. Although resorption rate is subject to individual variability and almost resorption occurs in 6 month after extraction, the alveolar ridge resorption persists for subsequent years to decades ${ }^{(13)}$. Available alveolar bone may be compromised in the in the posterior maxillae may be compromised because of sinus pneumatization and/or residual ridge resorption after tooth loss. The average height of the available bone in the edentulous maxilla was classified into three classes ${ }^{(11)}$.

\section{Short implants: design, and clinical aspects}

The rehabilitation treatment by implants instituted new concepts of dental prosthesis planning, and this approach provided an efficient masticatory function to patient as well as established aesthetical alternatives $^{(14)}$. Despite this, such rehabilitative strategy demands the possibility of osseointegrated implant placement into the remaining tooth socket and/or basal bone ${ }^{(15)}$. Otherwise, implant-supported prosthesis planning will be limited, requiring other treatment options to satisfy patient's need ${ }^{(14)}$. The pattern of bone loss after tooth extraction at maxilla's posterior area is important to understand implant position; Maxilla presents a greater horizontal loss, at buccal-palatal direction, with a slow vertical 
loss ${ }^{(16)}$. Maxilla's vertical bone loss occurs in two directions - the natural height remodeling undergone by the crestal bone and maxillary sinus pneumatization $^{(16)}$. Because of this type of bone loss and the presence of important anatomical areas, the planning of atrophic arches' posterior sites is normally more complex ${ }^{(17)}$.

However, the posterior maxilla presents a uniquely challenging site for implant placement due to several complicating factors. Some of the factors that lead to difficulties in implant placement and success in the maxillary molar region are the following:

- Difficult and challenging access

- Commonly reduced interarch space

- Limited visibility

- Post extraction resorption that leads to extensive tissue loss over time, and sinus pneumatization

- Poor (type IV) bone quality associated with the least favorable success rates ${ }^{(18)}$.

\section{* Implants classification:}

- The type of fixture (subperiosteal, root from, hollow)

- $\quad$ The surface (machined, rough)

- The shape (straight, tapered)

- The implant system (Branemark, Nobel Replac Straumann, Astra, 3i, MIS and bicon)

- Diameter (wide, regular, narrow)

- Length(long and short implant $)^{(19)}$.

\section{AIM OF THE STUDY}

The aim of this study was to evaluate clinically and radiographically the outcomes of using short implants as an alternative for sinus lift in extreme atrophic maxilla.

\section{PATIENTS AND METHODS}

Partially edentulous patients of both sexes (mean age 31.5 years, range 27.0-55.3 years, seven women and five men) were selected from the out-patient clinic, Faculty of Oral and Dental Medicine, Boys, Cairo, Al-Azhar University.

Grouping: - The patients were divided into two groups:

Group (I) the patients were treated with dental short implant placement without sinus lift, (eight implants with short length $5 \mathrm{~mm}$ ).

Group (II) the patients were treated with dental implant placement after sinus lift, (four implants with long length which higher than bone 2-3 mm) according to case.

- Patient examination (past medical history, past dental history , chief complaint).

- Radiographic examination (by panoramic X-ray \& Cone Bram C.T.Presurgery and Postsurgery $0,3,6$ months).

- Surgical procedures for implant installation. A pyramidal muco-periosteal flap was performed in the prepared surgical site area using Bard Parker No.15 surgical blade. The crestal incision started $1 / 2-\mathrm{cm}$ distal to pre-planned fixture position toward palatal side and end 1-cm mesial then two semivertical incisions descended buccally and the muco-Periosteal flap was elevated using periosteal elevator. The black silk 3-0 was used to invert flap and fixed it away from bone then large round surgical bur (no. 6) rotating at very low-speed was introduced at the prospective implant site in the maxilla to create bony concavity which is necessary for stable direction control of Pilot drill. Then drilling was started using Standard Pilot drill then $2.0 \mathrm{~mm}$ diameter drill that was held parallel the long axis of teeth then moved up and down during drilling with light intermittent finger pressure with internal irrigation up to level equal implant 
length. Pin $0^{\circ}$ was held in drilling site to detect its Paralleling direction with long axis of remaining teeth. Enlargement of the drilling site was made by using $2.5 \mathrm{~mm}$ diameter Standard Latch Reamer followed by $3 \mathrm{~mm}$ even $4.5 \mathrm{~mm}$ diameter Standard Latch Reamer, Enlargement of the drilling site was made by using $5.0 \mathrm{~mm}$ diameter hand reamer. The implant with its fixture mount was removed from its sterile package and handled to its position inside the prepared site. The implant was slowly threaded into the bone using ratchet or handle instrument by a clockwise direction under external saline irrigation. The fixture mount was then removed from the top of the implant by the anti- clockwise direction then cut in suitable length and goes back to its position. Topical ointment antibiotic applied, then fixture was covered after black retraction suture was removed, then thorough debridement and irrigation of the surgical field with sterile normal saline solution. The flap was repositioned and sutured using 3-0 vicryl suture material. Three month postoperatively, implant load by final abutment and cement with fixed prostheses.

\section{Methods of evaluation:}

A- Mobility: All implants were tested for proper osseointegration ,function and absence of mobility at three and six months after surgery .

B- Gingival index: The condition of the gingival tissues around the fixture abutments was evaluated according to Loe and Sillness .

\section{C- Radiographic Evaluation}

(A) Bone Density Measurements (densitometry analysis).

(B) Crestal bone resorption.

\section{Methodology}

All subjects of the study were submitted to Cone Beam scanning before implant placement, another cone beam C.T. was taken immediately after placement, after 3 months and after 6 months.

\section{RESULTS}

The mean value of crestal bone loss was 0.29 $\mathrm{mm}$, with a maximum of $1.77 \mathrm{~mm}$. No significant differences were found in the rate of bone thickness loss between group (I) and group (II) ( $\mathrm{P}=0.191)$. The mean value of bone loss increased in longer implants, but the difference was not significant $(\mathrm{P}=0.313)$. Spearman's correlation coefficient was 0.039 between the mean value of bone loss and implant length $(\mathrm{P}=0.717)$ and 0.242 between the patients' age and implant length $(\mathrm{P}=0.068)$. According to the survival criteria employed in the present study, failure did not occur in any of the participants of group (I) and group (II), so the overall survival rate was $100 \%$ for both groups. Clinically, the whole period of the investigation, implant mobility, recurrent peri-implant infection and radiolucency was not observed around the implants. None of the patients complained of continuous pain.

\section{DISCUSSION}

One of the difficulties encountered when evaluating short implant studies is the subjectivity of the term "short" implants. However, there is still no consensus in the literature on the definition of a short implant ${ }^{(21)}$. There were a big controversy about this definition concept (from 1990 - up till now). These differences must be considered for an adequate evaluation and comparison between the studies ${ }^{(3,8,14)}$ considering short implants include those which were 11,12 , and $13 \mathrm{~mm}$ long. Others authors $^{(6,29)}$ defined an implant length of $10 \mathrm{~mm}$ also as a short implant. However, Bernard et al $(1995)^{(21)}$ considered any implant less $10 \mathrm{~mm}$ in length as short ${ }^{(21)}$, while few studies have included that short implants $8 \mathrm{~mm}$ long or less ${ }^{(22)}$. With complete respect to previous structured reviews, this study may be appropriated to define a short implant as a device with a designed intra-bony length of $8 \mathrm{~mm}$ long or less. It was illustrated that unlike what occurs with the stresses applied to a natural tooth and the periodontal ligament, stresses around 
implants are greatest at the crest of the ridge and less in the apical portion ${ }^{(2)}$. Based on this principle, an increased length would simply improve primary stability of the implant during initial placement and enhance osseointegration $^{(22)}$. On the other hand, a wider diameter implant would increase not only primary stability but also the functional surface area at the crestal bone level, and thus lead to a better distribution of occlusal forces to the surrounding bone $^{(22)}$.

Most studies on short implants have reported that these implants can be restored with any type of prosthesis (i.e. single crowns, fixed partial denture, and removable prostheses) ${ }^{(23)}$. However, crownto-implant ratio, excessive occlusal forces, and presence of cantilevers are some of the risk factors that may lead to an increased stress on the implants and may therefore compromise implant survival ${ }^{(4)}$. Severe forms of ridge resorption are viewed to be beyond the scope of implant prosthodontics. A minimum of eight $\mathrm{mm}$ clearance from the sinus wall was considered essential for implant provision. Unless this space is evident, augmentation and grafting procedures were considered compulsory requirements. Seeking alternatives to the time consuming and risky augmentation procedures became a motivating topic for researches. The reliance to use short implants was raised after the finding of the phenomenon of the "crestal load focus". It was shown that when implants are loaded, most stresses are concentrated within the crestal 7 to $9 \mathrm{~mm}$ of the bony ridge ${ }^{(24)}$.

According to the current study, badly resorbed ridges can be served by means of short implant supported single tooth.Dentulous patients were selected to limit the functional loads transmitted to short implants as the magnitude of biting force changes in accordance to the state of dentation. Therefore, short implants were viewed to be a less critical option with the edentulous jaws as it is with dentulous patients ${ }^{(24)}$. Aside from the routine surgical protocol of implant placement, two surgical precautions were overemphasized. The first is to apply the bone expansion concept during osteotomy procedure. This augments the initial implant stability. The second precaution is to slightly over sink the implant into its foundation. This provides a more secure implant house far away from the overlying stresses transmitted throughout the mucosal layer ${ }^{(24)}$. Radiographic evaluation of the bony outlay (density and length) of the implants did not show any significant difference in bone loss associated with the different implant group numbers used .This finding seems to be inconsistent with clinical studies on Implants products where the survival rates for shorter implants $(8-9 \mathrm{~mm})$ were similar to longer implants $(\geq 10 \mathrm{~mm})^{(5)}$.

In the last decade, studies revealed conflicting results concerning the long-term survival and success rates of short implants ${ }^{(3)}$ which classified into following groups accord to percentage of success rate: In $1^{\text {st }}$ group of studies comparing survival rates of short implants, mostly machined-surface, with longer implants, failures were more often observed on implants of short length placed in maxillary sites where bone is generally of lesser density and the functional load remarkably increased. Failure rates (nearly 25\%). The worst results with short implants was documented for shorter implants by Winkler et $\mathrm{al}^{(64)}$. with an overall survival rate of $\mathbf{7 4 . 4 \%}$ for short implants (of the 43 implants placed, 11 were lost) and superior failure rate have been documented by Wyatt and Zarb (2010) $)^{(26)}$ with an overall survival rate of $\mathbf{7 5 \%}$ for short implants (of the 12 implants placed, three were lost). A second group of authors, although concluding that failure rates are higher with short implants, but it showed adequate survival rates ( up to 95\%) ${ }^{(25)}$. An earlier report, published in 1998 by ten Bruggenkate et al, reported on 253 short implants, with the investigators finding a survival rate of $(\mathbf{9 4 \%})$. Also, there were studies (Van Steenberghe et al. 1990; Jemt et al. 1995; Friberg et al. 1991) revealed adequate success rates of short implants after 1 to 5 years of follow 
up, ranging ( from 90 to $94.7 \%$ ) $^{(27)}$. A third group of authors which focused specifically on short implants, showed similar clinical outcomes to those reported in longer implants with survival rates ${ }^{(25)}$.

Friberg et al. also found success rate for short implants, with a $\mathbf{9 5 . 5 \%}$ five-year survival rate ${ }^{(22)}$. Also, Tawil and Younan observed 262 machined surface implants of $10 \mathrm{~mm}$ or smaller, which supported 163 prostheses. These authors obtained a excellent final success of $\mathbf{( 9 8 . 5 \% )}$ ) in cases employing short implants ${ }^{(3)}$. In 2009, retrospective study by Grant et al. involved 335 implants $8 \mathrm{~mm}$ in length placed in 124 patients (median age 56 years and 112 partially edentulous) between May 2005 and June 2007. The majority received fixed prostheses, while the remaining subjects received individual restorations. Four implants (in two patients) failed to osseointegrate, and one implant fractured. Of the remaining 330, for up to two years post-placement the survival rate was $(\mathbf{9 9 \%})^{(23)}$. Mario Santagat et al (2012) found total of 25 implants of $8 \mathrm{~mm}$ in length were placed with primary stability in 11 patients. Cumulative survival rates for implants were $100 \%{ }^{(24)}$. A fourth group of authors reported that implant length was not reported as influencing the survival rate (Buser et al. 1997; Ellegaard et al. 1997; Gunne et al. 1999; Brocard et al. 2000; Testori et al. 2001; Stellingsma et al. 2003; Feldman et al. 2004; Romeo et al. 2004; Lemmerman \& Lemmerman 2005) ${ }^{(3)}$.

Finally, It is widely agreed upon that the use of short implants would be better in cases of severely atrophic mandibles and/or pneumatization of the maxillar sinus, due to the fact that if a standard implant were to be inserted it would lead to a more invasive, expensive, and complex surgery (i.e., sinus lift, bone grafting procedures) ${ }^{(25)}$. Rokni et al. evaluated 199 implants, taking into account short implants and long implants. Long implants showed a greater bone loss of the alveolar bridge in relation to short implants ${ }^{(26)}$.
In the current study, all short implant which used in atrophic maxilla without sinus left and long length implant with sinus left were success and survival rates for all implants after insertion for six month were $100 \%$, this result combatable with report of $3^{\text {rd }}$ group authors. We can explain historical high failure survival rate of short implants compared with long implant in the posterior areas of maxilla by $1^{\text {st }}$ group authors as proposed by Telleman et $\mathrm{al}^{(27)}$. First, there was less area of contact between bone and implant. Second, most implants types with machine surface. And third, poor bone quality of maxilla. While the results improved in next groups of studies as mentioned due to increase area of contact this determined by 4 factors: the length, diameter, taper, and texture of the implant surface. However, short implants' length can be compensated by wider diameter (Currently available implants vary in diameter from 3 to $7 \mathrm{~mm}$ ), presence or absence of threads, the distance between the threads (thread pitch) and the depth of the threads, additional macroirregularities, and the shape/outline of the implant's body constitutes important aspects of its design ${ }^{(26)}$. Finite element studies suggest on implant with a wider diameter is more favorable in reducing the stress distribution in bone surrounding the implants. And where the bone quality is Poor, The retention of the wider diameter implants was mainly at the cortical layer which had a thin thickness of $0.5 \mathrm{~mm}$, whereas the rest of the long implant length was in the cancellous bone which had a low stiffness, causing a high deformation range. Also, from a biomechanical standpoint, the use of wider diameter implants allows engagement of a maximal amount of bone and improved distribution of stress in the surrounding bone. The use of wider components also allows for the application of higher torque in the placement of prosthetic components ${ }^{(28)}$.

\section{CONCLUSION}

Based on the result of this study it can be stated the following: 
I) Short implants' is successful treatment modality to avoid invasive surgical treatments at atrophic areas of posterior maxilla, such as bone grafts and sinus lifting.

II) The reconstruction of edentulous, atrophic jaws according to functional and aesthetic factors not only restores chewing function, but leads to positive psychosocial effect and thus also improves the patient quality of life.

\section{ACKNOWLEDGEMENT}

My special gratitude and deep appreciation are presented to Dr. Ahmed El-fekey for his helpful directions, honest assistance and valuable advices throughout the course of this thesis.

\section{REFERENCES}

1. Berglundh T., Persson L., and Klinge B., "A systematic review of the incidence of biological and technical complications in implant dentistry reported in prospective longitudinal studies of at least 5 years," Journal of Clinical Periodontology, vol. 29, supplement 3,pp. 197-212, 2002.

2. Misch C. E., Steigenga J., Barboza E., Misch-Dietsh F., and Cianciola L. J., "Short dental implants in posterior partial edentulism: a multicenter retrospective 6-year case series study," Journal of Periodontology, vol. 77, no. 8, pp. 1340-1347, 2006.

3. Tawil G, Younan R. Clinical evaluation of short, machined-surface implants followed for 12 to 92 months. Int J Oral Maxillofac Implants 2003;18(6):894-901 .

4. Fugazzotto PA. Shorter implants in clinical practice: rationale and treatment results. Int J Oral Maxillofac Implants 2008;23(3):487-96

5. Degidi M, Daprile G, Piattelli A. Primary stability determination by means of insertion torque and RFA in a sample of 4,135 implants. Clin Impl Dent Rel Res 2012;14(4):501-07

6. Jivraj S, Chee W, Corrado P. Treatment planning of the edentulous maxilla. Br Dent J. 2006;201:261-279; quiz 304.

7. Khojasteh A, Sadr SJ, Saboury A, Shidfar S. Onlay bone augmentation and bilateral open sinus lifting with simultaneous implant placement in a cherubic patient. J Craniofac Surg. 2014;25:e193-e196.
8. Boyne PJ, James RA. Grafting of the maxillary sinus floor with autogenous marrow and bone. J Oral Surg. 1980; 38:613-616

9. Summers RB. A new concept in maxillary implant surgery: the osteotome technique. Compendium.1994;15:152, 154-156, 158 passim; quiz 162.

10. Pjetursson BE, Tan WC. A systematic review of the success of sinus floor elevation and survival of implants inserted in combination with sinus floor elevation. J Clin Periodontol. 2008;35:216-240.

11. Nimigean V, Nimigean VR, Măru N, Sălăvăstru DI, Bădiţă D, Tuculină MJ. The maxillary sinus floor in the oral implantology. Rom J Morphol Embryol. 2008;49:485-489.

12. Sharan A, Madjar D. Maxillary sinus pneumatization following extractions: a radiographic study. Int J Oral Maxillofac Implants. 2008;23:48-56.

13. Tallgren A. The continuing reduction of the residual alveolar ridges in complete denture wearers: A mixedlongitudinal study covering 25 years (Reprinted from J Prosthet Dent vol 27, pg 120-32, 1972) J Prosthet Dent. 2003;89:427-435.

14. Laufer BZ, Gross M. Splinting osseointegrated implants and natural teeth in rehabilitation of partially edentulous patients. Part II: principles and applications. J Oral Rehabil. 1998 Jan;25(1):69-80

15. Buser D, Ingimarsson S, Dula K, Lussi A, Hirt HP, Belser UC. Long-term stability of osseointegrated implants in augmented bone: a 5-year prospective study in partially edentulous patients. Int J Periodont Rest Dent. 2002; 22:109-17.

16. Chiapasco M, Zaniboni M, Rimondini L. Autogenous onlay bone grafts vs. alveolar distraction osteogenesis for the correction of vertically deficient edentulous ridges: a 2-4-year prospective study on humans. Clin Oral Implants Res. 2007 Aug; 4:432-40.

17. Graves DT, Liu R, Alikhani M, Al-Mashat H, Trackman PC. Diabetes-enhanced inflammation and apoptosis: impact on periodontal pathology. J Dent Res. 2006 Jan; 85(1):15-21.

18. Koo KT, Wikesj UM, Park JY, Kim TI, Seol YJ, Ku Y et al. Evaluation of single-tooth implants in the second molar region a 5-year life-table analysis of a retrospective study. J Periodontol. 2010 Sep;81(9):1242-1249.

19. Marianne M, et al. The challenge of implant therapy in the posterior maxilla: Providing a rationale for the use of short implants. J Oral Implantol. 2007; 33(5):257-266. 
20. Hao Y, Zhao W, Wang Y, Yu J, and Zou D. Assessments of jaw bone density at implant sites using 3D cone-beam computed tomography. Eur Rev Med Pharmacol Sci 2014; 18: 1398-1403.

21. Bernard, J.P., Belser, U., Szmuckler, S., Martinet, J.P., Attieh, AS, ITI de faible longueur dans les secteurs postérieurs: résultats d'une étude clinique a 3 ans. Médecine Buccale, Chirurgie Buccale 1995; 1: 1-18.

22. Tutak M, et al. Short dental implants in reduced alveolar bone height: A review of the literature. Med Sci Monit, 2013; 19: 1037-1042.

23. Gentile MA, Chuang SK, Dobson T. Survival estimates and risk factors for failure with 6 X 5.7-mm implants. Int J Oral Maxillofac Implants 2005;20:930-937.

24. Raviv E. et al. Short dental implants in reduced alveolar bone height.Quintessence Int J 2010;41:575-579.

25. Tawil P, Tawil G. Short implants in deficient posterior jaws: current knowledge. Journal of the Lebanese Dental Association 2009;46(1):9-16

26. Wyatt, C.C.L. \& Zarb, G.A. Treatment outcomes of patients with implant-supported fixed partial prostheses. Int J Oral Maxillofac Impl 1998; 13: 204-211.

27. Jamal Hassan Assaf, et al. Short implants with single-unit restorations in posterior regions with reduced height $-\mathrm{a}$ retrospective study. Braz J Oral Sci, 2010; 9(4):493-497.
28. Ten Bruggenkate CM, Asikainen P, Foitzik C, Krekeler G, Sutter F. Short (6 mm) nonsubmerged dental implants: Results of a multicenter clinical trial of 1 to 7 years. Int J Oral Maxillofac Implants 1998;!3: 791-798.

29. Grant BT., Pancko FX. and Kraut RA. Outcomes of placing short dental implants in the posterior mandible: a retrospective study of 124 cases. J Oral Maxillofac Surg 2009;67(4):713-717.

30. Santagata M., et al. Augmentation of atrophic posterior maxilla by short implants and osteotome technique . Stomatologija, Baltic Dent Maxillofac J 2012; 14(3) : 85-88.

31. Diego Lops et al. Short Implants in Partially Edentulous Maxillae and Mandibles: A 10 to 20 Years Retrospective Evaluation. Int J Dent 2012, Article ID 351793:1-8.

32. Chizolini et al. Short implants in oral rehabilitation: Literature Review Article. RSBO,2011 Jul-Sep;8(3):329-34

33. Telleman G, Raghoebar GM, Vissink A, et al. A systematic review of the prognosis of short $(, 10 \mathrm{~mm})$ dental implants placed in the partially edentulous patient. J Clin Periodontol. 2011; 38:667-676.

34. 34. Eitan Mijiritsky, et al. Implant diameter and length influence on survival: interim results during the first 2 years of function of implants by a single manufacturer. J impl Dent, 2013;22(4):394-398. 